\title{
Prospective evaluation of endoscopic ultrasonography and microscopic examination of duodenal bile in the diagnosis of cholecystolithiasis in 45 patients with normal conventional ultrasonography
}

\author{
P Dahan, C Andant, P Lévy, P Amouyal, G Amouyal, M Dumont, S Erlinger, \\ A Sauvanet, J Belghiti, M Zins, V Vilgrain, P Bernades
}

\begin{abstract}
The aim of this study was to prospectively evaluate endoscopic ultrasonography and microscopic examination of duodenal bile in the diagnosis of cholecystolithiasis not detected by conventional ultrasonography. Forty five consecutive patients (26 females, 19 males, mean age: 50 years) with suspected cholecystolithiasis and at least two normal transcutaneous ultrasonography examinations were included. Endoscopic ultrasonographic criteria for the diagnosis of cholecystolithiasis were the presence of stones with or without acoustic shadowing or sludge. Criteria of microscopic examination of bile were cholesterol or bilirubinate crystals or spheroliths. Thirty three patients underwent cholecystectomy and lithiasis was found in gall bladder bile in 24. Twelve patients who were not operated on and were followed up (median: 17 months), had no evidence of cholecystolithiasis. Endoscopic ultrasonography and duodenal bile examination were $96 \%$ and $67 \%$ sensitive, respectively $(\mathbf{p}<0.03)$. The specificity was not different (86 and $91 \%$, respectively). None of the 16 patients with negative results in both procedures had evidence of cholecystolithiasis. It was found that for the diagnosis of cholecystolithiasis in patients with normal conventional ultrasonography, the sensitivity of endoscopic ultrasonography is higher than that of microscopic examination of duodenal bile. If endoscopic ultrasonography and microscopic examination of duodenal bile are negative, the risk of underdiagnosing cholecystolithiasis is negligible.

(Gut 1996; 38: 277-281)
\end{abstract}

Keywords: gall bladder, lithiasis, bile, endoscopic ultrasonography, microscopic bile examination.

and Service de

M Zins

V Vilgrain

Hôpital Beaujon, Clichy, France

Correspondence to: Dr P Lévy, Service de Gastroentérologie, Hôpital Beaujon, 92118 Clichy Cedex, France.

Accepted for publication 12 July 1995

The diagnosis of cholecystolithiasis is mainly based on conventional transcutaneous ultrasonography (TUS). ${ }^{12}$ TUS fails to detect cholecystolithiasis in some cases; it has been suggested that the detection of cholesterol or bilirubinate crystals in gall bladder or duodenal bile could identify patients with and those without cholecystolithiasis. ${ }^{3-6}$ The sensitivity of microscopic bile examination (MBE) is probably lower when duodenal bile is studied because of dilution with gastric, pancreatic, and intestinal secretions and possible incomplete contraction of gall bladder even after injection of caeruleine. 57

Endoscopic ultrasonography (EUS), a new imaging procedure, is particularly useful for exploring the biliopancreatic region and has a high sensitivity for the diagnosis of choledocholitiasis. $^{89}$ The usefulness of EUS for the diagnosis of cholecystolithiasis has not been assessed. The aim of this study was to prospectively evaluate and compare the accuracy of EUS and MBE of gall bladder bile collected in the duodenum in consecutive patients suspected of having a cholecystolithiasis but with negative TUS.

\section{Methods}

\section{Selection of patients}

From January 1992 to July 1993, all patients who fulfilled the inclusion criteria were prospectively studied. Inclusion criteria were either (a) transient epigastric or right upper quadrant pain with fever and jaundice associated with raised serum alkaline phosphatase activities or raised serum $\gamma$-glutamyltransferase or transaminase activities, or both over two times the upper limit of normal $(n=20)$, or (b) acute pancreatitis defined by acute epigastric pain requiring hospitalisation associated with increased serum amylase or lipase activities over three times the upper limit of normal $(n=25)$. Alcoholic patients and patients suspected of having acute pancreatitis from other causes (drugs, hypercalcaemia, hypertriglyceridaemia) were excluded. Patients must also have had at least two normal TUS examinations (mean $2 \cdot 3$, range: $2-4$ ) with at least one performed in our institution by an experienced radiologist. Patients with abnormal gall bladder (stones, sludge, thickened wall) or with abnormal common bile duct (stones, sludge, diameter enlargement, thickened wall) were excluded.

\section{Interventions}

In our institution, TUS was performed with real time, Siemens Sonoline (SL2) or 
Komtron Signa 1 (frequency: 3.5 and 5 $\mathrm{MHz}$ ). To increase TUS sensitivity, patient position was changed during the examination (to mobilise gall bladder lithiasis) and patients were given water to reduce intestinal gas. TUS diagnostic criterion for gall bladder lithiasis was a hyperechoic structure within the gall bladder sometimes associated with an acoustic shadow.

All patients included underwent EUS and MBE of gall bladder bile collected from the duodenum. EUS was performed within 72 hours after the last TUS with an Olympus GFUM 3/EUM 3 or GFUM 20/EUM 20. The transducer was inserted into the inferior portion of the second duodenum and gradually drawn back to the stomach. The acoustic liaison between the transducer and the digestive wall was achieved by a balloon filled with 5-20 ml water. The gall bladder was examined through the duodenum and the stomach depending on anatomical variations using $7 \cdot 5 \mathrm{MHz}$ transducer frequency. The examination lasted 10-30 minutes. Patients were sedated with intravenous propofol (ICI Pharma, Cergy, France). The gall bladder was examined in all cases. Images were interpreted by four skilled operators (PD, PL, GA, PA) unaware of the MBE results.

EUS diagnostic criteria for gall bladder lithiasis was the presence within the gall bladder of: (a) a hyperechoic semicircular arch or circular shape associated with an acoustic shadow (Fig 1); (b) a hyperechoic foci without associated acoustic shadowing (Fig 2); (c) gall bladder sludge defined by mobile, low amplitude echoes seen in the lumen that layered the most dependent part of the gall bladder without associated acoustic shadowing ${ }^{10}$ (Fig 3).

Collection of duodenal bile was performed under endoscopic control. A polyvinyl single holed catheter $(2.2 \mathrm{~mm}$ diameter; Olympus) was introduced into the operating channel of the echoendoscope or endoscope (Olympus GIF XQ 20) and placed in the second duodenum near the papilla of Vater, 10 to 15 minutes after intramuscular injection of $30 \mu \mathrm{g}$ of caerulein (Cerulex, Farmitalia Carlo Erba, Rueil Malmaison, France). Two to four two millilitre aliquots of bile were aspirated from the catheter by gentle suction through a sterile syringe and the darkest aliquot was used for microscopic examination. Bile samples were kept at $37^{\circ} \mathrm{C}$ for at least one hour and then centrifuged at $12000 \mathrm{rpm}$ for five minutes. A drop of bile was examined under direct and polarised light microscopy. All manipulations were performed in sterile glass tubes prewarmed to $37^{\circ} \mathrm{C}$. MBE was performed immediately, 24 hours, and 48 hours after collection while bile samples were kept at $37^{\circ} \mathrm{C}$. The diagnosis of cholecystolithiasis was considered if at least one typical cholesterol crystal or bilirubinate granule were present according to criteria established by Juniper and Burson ${ }^{3}$ or in the presence of a large amount of spheroliths. ${ }^{11}$ Duodenal MBE was always performed after EUS without knowledge of EUS results.

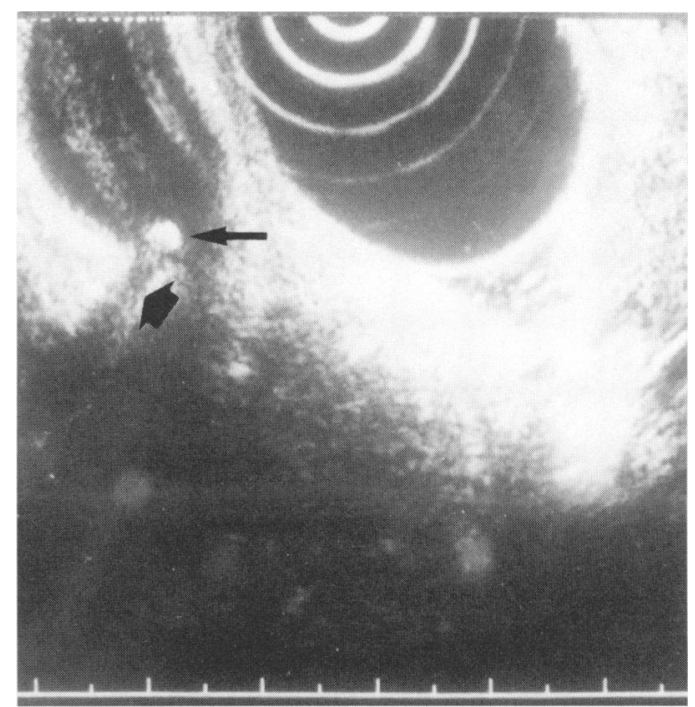

Figure 1: Transduodenal endoscopic ultrasonography: hyperechoic circular shape (thin black arrow) with a $3 \mathrm{~mm}$ diameter associated with an acoustic shadow (thick black arrow) suggesting a stone in the gall bladder infundibulum.

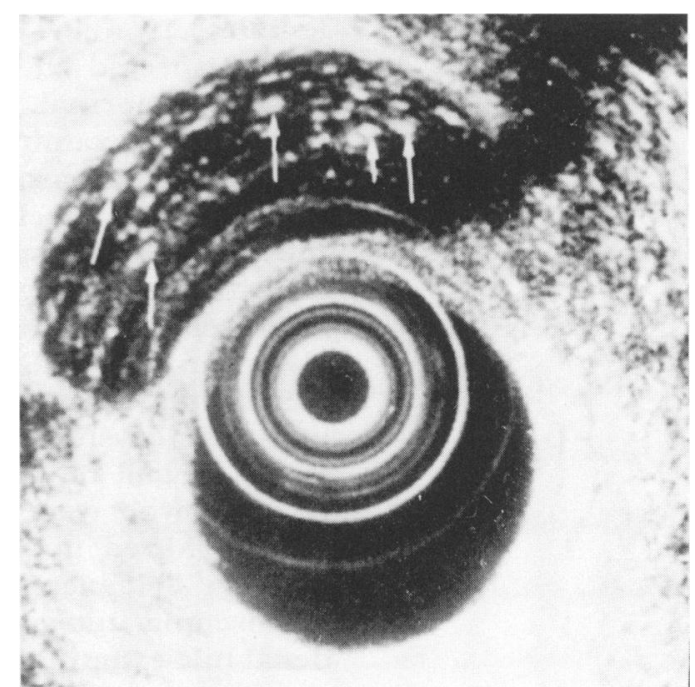

Figure 2: Transduodenal endoscopic ultrasonography: hyperechoic foci with a diameter $<2 \mathrm{~mm}$ without associated acoustic shadowing suggesting stones (white arrows).

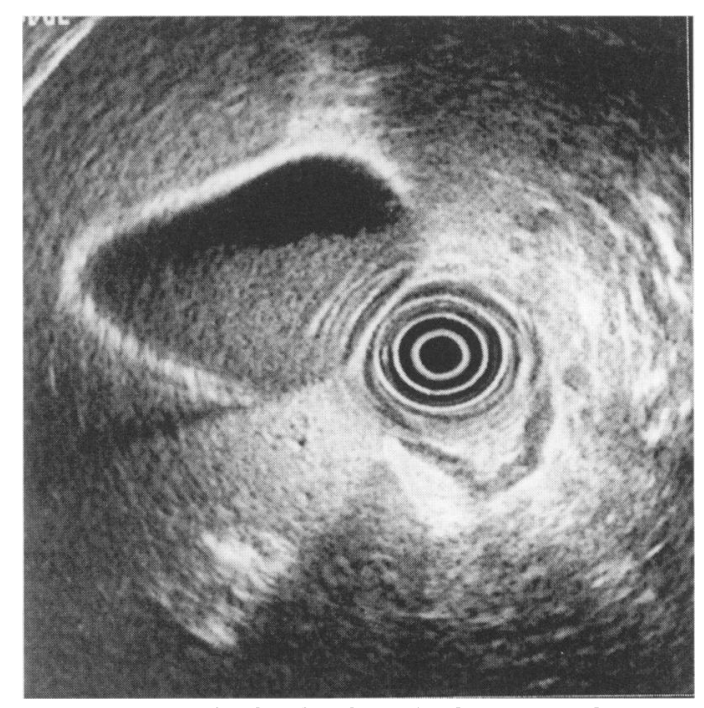

Figure 3: Transduodenal endoscopic ultrasonography: mobile, low amplitude echoes seen in the lumen, which layered in the most dependent part of the gall bladder without associated acoustic shadowing suggesting gall bladder sludge. 
TABLE I Patient characteristics

\begin{tabular}{llll}
\hline Clinical symptoms & Number & $\begin{array}{l}\text { Women/ } \\
\text { men }\end{array}$ & $\begin{array}{l}\text { Age (y) } \\
\text { (median (range)) }\end{array}$ \\
\hline $\begin{array}{l}\text { Acute pancreatitis } \\
\begin{array}{l}\text { Symptoms of } \\
\text { cholecystolithiasis }\end{array}\end{array}$ & 25 & $14 / 11$ & $49(28-66)$ \\
\begin{tabular}{l} 
Total \\
\hline
\end{tabular} & 45 & $12 / 8$ & $52(24-78)$ \\
\hline
\end{tabular}

\section{Therapeutic decision}

A laparoscopic or traditional cholecystectomy was performed: (a) if EUS or MBE suggested the presence of lithiasis, (b) in patients without radiological, duodenal MBE and EUS abnormalities but with clinical and biochemical data strongly suggesting lithiasis without any other obvious causes; in these patients, the decision of a cholecystectomy was made by the clinician responsible for the patient.

In patients who were operated on, gall bladder bile was collected operatively by needle puncture and then immediately filtered through a pad to look for macroscopically visible stones. A MBE was performed with the same methods as above. The gall bladder was then opened and macroscopically visible stones were searched for. Gall bladder lithiasis was diagnosed if macroscopically visible stones or typical cholesterol crystals or bilirubinate granules were present according to criteria established by Juniper and Burson ${ }^{3}$ or if there were many spheroliths. ${ }^{11}$

Patients who did not undergo cholecystectomy were followed up for at least six months and serum transaminase, alkaline phosphatase, and amylase activities were measured every six months. These patients were interviewed for clinical signs of biliary disease. The decision to repeat TUS was made by the clinician responsible for the patient. All non-operated patients were contacted in June 1994.

\section{Statistical methods}

Both procedures (EUS and duodenal MBE) were compared using conventional $\chi^{2}$ test with Yates's correction to study variations in predictability. A p value $<0.05$ was considered significant. Sensitivity and specificity as well as positive and negative predictive values were calculated within the selected patient groups.

Results

Table I gives the main characteristics of 45 patients ( 26 women, 19 men; median age: 50 (range 24-78) years). The mean time between EUS and bile sampling was 2.7 days (range $0-32$ ). There were no procedure related complications.

\section{EUS results}

Results of EUS suggested cholecystolithiasis in 26 patients (Table II). None of the patients had direct or indirect signs of stones in the common bile duct. The 26 patients with positive EUS were operated on and the presence of lithiasis markers was confirmed at cholecystectomy in 23 including 17 patients with macroscopically visible stone. Table II shows the correlation between EUS results, duodenal MBE, and that of the examination of operatively obtained bile. The diagnosis was not confirmed by the examination of operatively obtained bile in three patients. In these, EUS only showed hyperechoic foci without acoustic shadowing after transcutaneous manual mobilisation of the gall bladder and the duodenal MBE was negative.

In 19 patients in whom EUS did not suggest cholecystolithiasis, seven underwent cholecystectomy (four based on the choice of the clinician, three because pre-operative duodenal MBE was positive). Lithiasis markers were found in only one of these patients (Table III). Table IV summarises the results of EUS.

\section{Duodenal MBE results}

Duodenal MBE was positive in 18 patients and showed cholesterol crystals in 14, bilirubinate granules in three, and spheroliths in one. All these patients were operated on and the presence of lithiasis markers was confirmed at cholecystectomy in 16 including nine with macroscopically visible stone. In two patients (one with spheroliths, one with cholesterol crystals), the diagnosis was not confirmed by examination of the operatively obtained bile.

In 27 patients in whom duodenal MBE was negative, 15 underwent cholecystectomy (11 because pre-operative EUS was positive, four based on the choice of the clinician). Macroscopically visible stones were found in eight patients and no lithiasis markers were found in the remaining patients. Tables II and III show the correlation between duodenal and operative MBE. Table IV summarises the results of duodenal MBE.

\section{Diagnostic and therapeutic procedure}

Thirty three patients underwent cholecystectomy, 29 because lithiasis criteria were present at EUS or duodenal MBE (EUS alone: $n=11$,

TABLE III Correlation between duodenal MBE and operative gall bladder bile examination in seven patients with negative EUS who were operated on

\begin{tabular}{ll}
\hline Duodenal $M B E$ & $\begin{array}{l}\text { Operative gall bladder bile } \\
\text { examination }\end{array}$ \\
\hline Cholesterol crystals $(\mathrm{n}=1)$ & Cholesterol crystals $(\mathrm{n}=1)$ \\
Cholesterol crystals $(\mathrm{n}=1)$ & No lithiasis $(\mathrm{n}=1)$ \\
Spheroliths $(\mathrm{n}=1)$ & No lithiasis $(\mathrm{n}=1)$ \\
No lithiasis $(\mathrm{n}=4)$ & No lithiasis $(\mathrm{n}=4)$ \\
\hline
\end{tabular}

$\begin{array}{cll}\begin{array}{c}\text { Hyperechoic foci without } \\ \text { acoustic shadowing } \\ (\mathrm{n}=18)\end{array} & \begin{array}{l}\text { No lithiasis }(\mathrm{n}=2) \\ \text { Cholesterol crystals }(\mathrm{n}=6)\end{array} & \begin{array}{l}\text { Macroscopically visible stones } \\ (\mathrm{n}=10)\end{array} \\ & \begin{array}{l}\text { Cholesterol crystals }(\mathrm{n}=3) \\ \text { Bilirubinate granules }(\mathrm{n}=1)\end{array} & \begin{array}{l}\text { Cholesterol crystals }(\mathrm{n}=3) \\ \text { Spheroliths (n=1) }\end{array} \\ & \begin{array}{l}\text { Bilirubinate granules }(\mathrm{n}=1) \\ \text { No lithiasis }(\mathrm{n}=3)\end{array} & \begin{array}{l}\text { Bilirubinate granules }(\mathrm{n}=1) \\ \text { No lithiasis (n=3) }\end{array} \\ & & \end{array}$

${ }^{\star}$ Diameter $<2 \mathrm{~mm}$ in all cases.

$$
(\mathrm{n}=10)
$$

Spheroliths $(n=1)$

No lithiasis $(n=3)$
Operative gall bladder

Macroscopically visible stones $(n=3)$

Hyperechoic foci with $(n=3)^{\star}$

Sludge $(n=5)$

Cholesterol crystals $(n=1)$

No lithiasis $(n=2)$

Bilirubinate granules $(n=1)$

Bilirubinate granules $(n=1)$ Macroscopically visible stones $(n=4)$

Correlation between EUS findings, duodenal MBE, and operative gall bladder

\footnotetext{
¿
} 
TABLE IV Summary of the results of EUS and duodenal MBE for the diagnosis of cholecystolithiasis in 45 patients suspected of having gall bladder stones undetectable by conventional TUS

\begin{tabular}{lllllll}
\hline & \multicolumn{2}{l}{ EUS } & & \multicolumn{2}{l}{ Duodenal MBE } \\
\cline { 2 - 3 } & Positive & Negative & & Positive & Negative \\
\hline Number & 26 & 19 & & 18 & 27 \\
Lithiasis markers $(\mathrm{n}=24)^{\star}$ & 23 & 1 & & 16 & 8 \\
Patients with macroscopically visible stones & 17 & 0 & & 9 & 8 \\
No lithiasis markers $(\mathrm{n}=21)$ & 3 & 18 & & 2 & 19
\end{tabular}

^Patients with either macroscopically visible stones, cholesterol crystals, bilirubinate granules or spheroliths in operatively obtained bile.

TABLE V Sensitivity, specificity, positive and negative predictive values of EUS and duodenal MBE for the diagnosis of cholecystolithiasis in 45 patients suspected of having gall bladder stones undetectable by conventional TUS

\begin{tabular}{llll}
\hline & EUS & MBE & $p$ Value \\
\hline Sensitivity & $96(88$ to 100$)$ & $67(48$ to 85$)$ & $0 \cdot 03$ \\
Specificity & $94^{\star}$ & $82^{\star}$ & NS \\
Positive predictive value & $86(71$ to 100$)$ & $91(79$ to 100$)$ & NS \\
Negative predictive value & $89(77$ to 100$)$ & $89(74$ to 100$)$ & NS \\
\hline
\end{tabular}

(95\% confidence intervals).

*Sensitivity of EUS and MBE for detecting patients with macroscopically visible stones.

TABLE VI Summary of the results of EUS and duodenal MBE for the diagnosis of cholecystolithiasis in 25 patients suspected of having biliary pancreatitis without gall bladder stones detectable by conventional TUS

\begin{tabular}{lllllll}
\hline & \multicolumn{2}{l}{ EUS } & & \multicolumn{2}{l}{ Duodenal MBE } \\
\cline { 2 - 3 } & Positive & Negative & & Positive & Negative \\
\hline Number & 15 & 10 & & 9 & 16 \\
Lithiasis markers $(\mathrm{n}=14)^{\star}$ & 14 & 0 & & 7 & 7 \\
Patients with macroscopically visible stones & 11 & 0 & & 6 & 5 \\
No lithiasis $(\mathrm{n}=11)$ & 1 & 10 & & 2 & 9
\end{tabular}

^Patients with either macroscopically visible stones, cholesterol crystals, bilirubinate granules or spheroliths in operatively obtained bile.

duodenal MBE alone: $\mathrm{n}=3$, both procedures: $\mathrm{n}=15$ ) and four patients with negative EUS and duodenal MBE because of the clinician's choice. Bile obtained during the operation showed lithiasis markers in 24 patients: macroscopically visible stones: $n=17$, cholesterol crystals; $n=4$, bilirubinate granules: $n=2$, spheroliths: $n=1$. No lithiasis markers were found at cholecystectomy in nine patients including the four with negative EUS and MBE findings. No patient had choledocholitiasis. Twelve patients were not operated on and were followed up (median: 17 (range: 12-21) months). All these patients were free of clinical and biochemical symptoms. Four of them also had a normal TUS during follow up period, 12-21 months after EUS and MBE.

\section{Comparison of EUS and duodenal $M B E$}

Table V shows the sensitivity and specificity, as well as positive and negative predictive values of the two procedures for the detection of cholecystolithiasis in the group of selected patients. EUS was more sensitive but as specific as duodenal MBE for the detection of cholecystolithiasis. In 16 cases, both EUS and duodenal MBE were negative. None of these 16 patients had evidence of cholecystolithiasis as shown by MBE of bile obtained at cholecystectomy in four patients and during follow up in 12 patients (four had a normal TUS during follow up). If the 12 patients who did not have a cholecystecomy are considered as false negatives, EUS and duodenal MBE sensitivity reaches $64 \%$ and $44 \%$, respectively.

\section{Results of EUS and MBE in the subgroup of}

patients with acute pancreatitis $(n=25)$

Table VI gives the EUS results. None of the patients with negative EUS had evidence of cholecystolithiasis based on examination of bile obtained at cholecystectomy in four and follow up in six. In this group of patients with suspected acute biliary pancreatitis, the sensitivity and specificity of EUS were $100 \%$ and $91 \%$, respectively.

Table VI gives the duodenal MBE results. Seven patients with negative duodenal MBE had evidence of lithiasis markers in the bile obtained at cholecystectomy (false negative) including five patients with macroscopically visible stones. In nine patients with negative MBE, there was no evidence of cholecystolithiasis based on examination of bile obtained at cholecystectomy in three and follow up in six.

In the group of patients with suspected acute biliary pancreatitis, the sensitivity of EUS and duodenal MBE were $100 \%$ and $50 \%$ (95\% confidence intervals: 24 to $76 \%$ ), respectively $(p<0.01)$. The specificity of the two procedures was $91 \%$ (95\% confidence intervals: 76 to $100 \%$ ) and $82 \%$ (95\% confidence intervals: 59 to $100 \%$ ), respectively (not significant).

\section{Discussion}

The aim of this study was to prospectively evaluate the accuracy of EUS in diagnosing cholecystolithiasis when conventional TUS is negative and to compare EUS to duodenal MBE. The diagnosis of cholecystolithiasis was considered highly probable if lithiasis markers - that is, macroscopically visible stones, cholesterol crystals, bilirubinate granules, spheroliths - were present in bile collected operatively. ${ }^{6}$ The sensitivity of EUS (96\%) was significantly higher than duodenal MBE (67\%) but the specificity of both techniques was similar ( $86 \%$ and $91 \%$, respectively). If we only considered macroscopically visible stones as the gold standard for the positive diagnosis of lithiasis, the sensitivity of EUS and MBE were 94 and $82 \%$, respectively.

The accuracy of TUS in detecting cholecystolithiasis is high. The sensitivity of TUS has ranged from 92 to $96 \%$ in previous studies. ${ }^{121213}$ TUS false negatives mainly result from (a) stones $<3 \mathrm{~mm}$ diameter that are below TUS resolution, (b) stones located in the gall bladder infudibulum that are difficult to visualise. In these cases, TUS sensitivity is about $65 \% .^{14}$ The specificity is about $100 \%$ if a hyperechoic image with acoustic shadowing is found in the gall bladder. ${ }^{12}$ New tools that are now available to explore the biliary tree may improve the detection rate of cholecystolithiasis in patients with symptoms suggesting gall stone disease.

Patients with negative EUS and duodenal MBE either had a cholecystectomy $(n=4)$ or were followed up $(n=12)$. A cholecystectomy was not justified for all patients in the study, 
particularly for those with clinical and biochemical data that did not sufficiently suggest gall stone disease. In these patients, the absence of gall stone disease was suggested by the absence of symptoms during follow up. As a result, we cannot be completely sure that these patients were free of gall stones. Nevertheless, lithiasis was not a clinical problem in these patients at least during the duration of follow up.

EUS was only shown to be falsely negative in one patient. This patient had a cholecystectomy because of positive duodenal MBE and microscopic cholesterol crystals were found at operation. EUS was falsely positive in three patients, but hyperechoic foci were only identified after manual transcutaneous mobilisation of the gall bladder. Perhaps the images seen in these cases were acoustic reverberation artefacts caused by gall bladder wall movement and thus, only 'spontaneously observed' hyperechoic foci that is, those seen without manual transcutaneous mobilisation - should be considered.

Many authors have shown that cholesterol crystals in bile are markers of the presence of stones. ${ }^{5-7}$ 15-17 Although the specificity of duodenal MBE was similar to that of previous reports, the sensitivity $(67 \%)$ was lower than in the studies by Ros et al (86\%), ${ }^{15}$ Buscail et al $(85 \%),{ }^{16}$ and Delchier et al $(88 \%) .{ }^{17}$ In these studies, bile collection was performed with direct cannulation of the papilla of Vater or with a catheter in the duodenum for 12 hours. The many duodenal MBE false negatives in this study were also found by other investigators $^{45}$ and may have many causes: (a) bile dilution by gastric, duodenal and pancreatic secretions: cholesterol crystals are less common in duodenal than in gall bladder bile $^{18}$; (b) the intermittent presence of cholesterol crystals in duodenal bile. ${ }^{7}$ Although several bile collections would certainly improve the sensitivity of this method, this would be less acceptable to patients. Two patients had false positive duodenal MBE. In one, MBE showed spheroliths. Spheroliths are only considered markers of gall stone disease when they are found in high numbers, ${ }^{15}$ a quantitative interpretation that may increase the variability of the results. There is no clear explanation for the other patient with false positive MBE showing cholesterol crystals.

In the 25 patients with suspected acute biliary pancreatitis, lithiasis markers were found in $14(56 \%)$. EUS sensitivity and specificity were $100 \%$ and $91 \%$, and those of duodenal MBE were $50 \%$ and $82 \%$, respectively. The diagnosis of cholecystolithiasis is particularly important and urgent as cholecystectomy or endoscopic sphincterotomy are required. In a study that included patients with acute pancreatitis, TUS sensitivity was $87 \% .^{11}$ In the study by Lee et al ${ }^{19}$ that included 31 patients with acute pancreatitis considered as idiopathic (no gall stones at TUS and no other causes), duodenal MBE showed bilirubinate granules or cholesterol crystals in $23(86 \%)$ but biliary sludge was shown by TUS in only $48 \%$. Our study showed that gall bladder sludge identified by EUS but not by TUS was also closely related to the presence of lithiasis markers. A previous study from our group showed that EUS was the most sensitive procedure for the diagnosis of choledocholithiasis. ${ }^{9}$ Thus, EUS may be performed in cases of acute pancreatitis of unknown aetiology especially if gall stones are suspected.

It is noteworthy that 16 patients with negative results for both EUS and MBE had no cholecystolithiasis as shown by cholecystectomy in four and follow up in 12. Therefore, the negative predictive value of both techniques may be $100 \%$. Associating these two procedures - which can be performed at the same time - may be a highly accurate method for diagnosing or excluding cholecystolithiasis. The clinical significance of this result is of particular importance in patients with pancreatitis of unknown aetiology and the drawbacks of undergoing EUS should be counterbalanced by the seriousness of failing to diagnose cholelithiasis.

The authors wish to thank Ms Dale Roche, editorial assistant of the fournal of Hepatology, for her help in preparing the manuscript.

1 Hessler PC, Hill DS, Defoirie FM, Rocco AA. High accuracy sonographic recognition of gallstones. $\mathrm{Am} \mathcal{f}$ Roentgenol 1981; 136: 517-20.

2 Cooperberg PL, Burhenne HJ. Real-time ultrasonography Diagnostic technique of choice in calculous gallbladder disease. $N$ Engl f Med 1980; 302: 1277-9.

3 Juniper K Jr, Burson EN Jr. Biliary tract studies. II. The significance of biliary crystals. Gastroenterology 1957; 32: 175-211.

4 Neoptolemos JP, Davidson BR, Winder AF, Vallance D. Role of duodenal crystal analysis in the investigation of idiopathic pancreatitis. Br f Surg 1988; 75: 450-3.

5 Abbas A, Baumann R, Schutz JF, Maillard D, Sondag D, Weil JP. Cristaux de cholestérol et lithiase biliaire. Intérêt de l'étude de la bile recueillie par tubage duodénal. Gastroenterol Clin Biol 1984; 8: 454-7.

6. Ramond MJ, Dumont M, Belghiti J, Erlinger S. Sensitivity and specificity of microscopic examination of gallbladder bile for gallstone recognition and identification. Gastroenterology 1988; 95: 1339-43.

7 Marks JW, Bonorris G. Intermittency of cholesterol crystals in duodenal bile from gallstone patients. Gastroenterology 1984; 87: 622-7.

8 Amouyal P, Palazzo L, Amouyal G, Ponsot P, Mompoint $\mathrm{D}$, Vilgrain V, et al. Endosonography: promising method for diagnosis of extrahepatic cholestasis. Lancet 1989; ii: for diagn

9 Amouyal P, Amouyal G, Lévy P, Tuzet S, Palazzo L, Vilgrain V, et al. Diagnosis of choledocolithiasis by endoscopic ultrasonography. Gastroenterology 1994; 106: 1062-7.

10 Conrad RR, Janes JO, Dietchy J. Significance of low level echoes within the gallbladder. Am f Roentgenol 1979; 132: 967-72.

11 Goodman AJ, Neoptolemos JP, Carr-Locke DL, Finlay DB, Fossard DP. Detection of gallstones after acute pancreatitis. Gut 1985; 26: 125-32.

12 Lee CL, Wu CH, Chen TK, Yang SS, Huang CS Prospective study of abdominal ultrasonography before laparoscopic cholecystectomy. $\mathcal{F}$ Clin Gastroenterol 1993; laparoscopic

13 McIntosch DMF, Pennay HF. Gray scale ultrasonography as a screening procedure in the detection of gallbladder disease. Radiology 1980; 136: 725-7.

$14 \mathrm{Kurol}$ M, Forsberg L. Ultrasonography in the diagnosis of cholecystitis. Acta Radiol 1984; 25: 379-83.

15 Ros E, Navarro S, Bru C, Garcia-Pugés A, Valderrama R. Occult microlithiasis in 'idiopathic' acute pancreatitis: prevention of relapses by cholecystectomy or ursodeoxycholic acid therapy. Gastroenterology 1991; 101: 1701-9.

16 Buscail L, Escourrou J, Delvaux M, Guimbaud R, Nicolet $\mathrm{T}$, Frexinos J, et al. Microscopic examination of bile directly collected during endoscopic cannulation of the papilla. Utility in patients with suspected microlithiasis. papilla. Utility in patients with

17 Delchier JC, Benfredj P, Préaux AM, Métreau JM, Dhumeaux D. The usefulness of microscopic bile examination in patients with suspected microlithiasis. Hepatology 1986; 6: 118-22.

18 Jonowitz P, Swobodnik W, Weschler JG, Zoller A, Kuhn K, Ditschuneit $\mathrm{H}$. Comparison of gallbladder bile and endoscopically obtained duodenal bile. Gut 1990; 31: 1407-10.

19 Lee SP, Nicholls JF, Park HZ. Biliary sludge as a cause of acute pancreatitis. N Engl f Med 1992; 326: 589-93. 